\title{
Site Characterization of the Northern Site of the Cherenkov Telescope Array
}

\author{
Markus Gaug ${ }^{1,2, \star}$, Lluís Font ${ }^{1,2}$, and Camilla Maggio ${ }^{1,2}$ \\ ${ }^{1}$ Unitat de Física de les Radiacions, Departament de Física, Universitat Autònoma de Barcelona, Spain \\ ${ }^{2}$ CERES-IEEC, Universitat Autònoma de Barcelona, Spain
}

\begin{abstract}
We present the current knowledge and activities to assess the climatology of the "Observatorio del Roque de los Muchachos" (ORM), selected to host the Northern observatory of the CTA, with particular emphasis on molecular density profiles and aerosol extinction.
\end{abstract}

\section{Introduction}

Recently, the site selection process for the Cherenkov Telescope Array (CTA) [1] has been resolved. For its Southern part (CTA-S), a plateau of the Cerro Armazones in Northern Chile, close to Paranal was chosen ${ }^{1}$, while the "Observatorio del Roque de los Muchachos" (ORM) on La Palma, Canary Islands, has been selected for its Northern observatory (CTA-N) ${ }^{2}$.

The ORM looks also back on a history of more than 20 years of gamma-ray astronomy through Imaging Atmospheric Cherenkov Telescopes (IACTs), featuring first the HEGRA ${ }^{3}$ and then the MAGIC Telescopes ${ }^{4}$. The latter dedicated considerable efforts to characterize the atmosphere above with respect to molecular and aerosol profiles, and to clouds [2-4]. Moreover, existing optical telescopes are active in this task [5, 6], and the very same host organization of the ORM, the "Instituto de Astrofísica de Canarias" (IAC), through their "Sky Quality Group" (SQG) ${ }^{5}$ [7-9]. In addition, also the "European Extremely Large Telescope" (E-ELT) have carried out site testing and characterization campaigns on La Palma [10]. Moreover, the only about $150 \mathrm{~km}$ apart "Observatorio del Teide" (OT $)^{6}$, and particularly the "Izaña Atmospheric Research Center" (IARC) ${ }^{7}$, or simply Izaña Observatory (IZO), both located on the neighboring island of Tenerife at about $2400 \mathrm{~m}$ a.s.1., have monitored atmospheric conditions over large time series. Atmospheric conditions between Tenerife and La Palma, at these altitudes, are highly correlated [11], and data from the IARC can hence be used, at least to better understand the aerosol characteristics, at the ORM.

\footnotetext{
^e-mail: markus.gaug@uab.cat

${ }^{1}$ see www.cta-observatory.org/about/array-locations/chile/

${ }^{2}$ see www.cta-observatory.org/about/array-locations/la-palma/

${ }^{3}$ see www.mpi-hd.mpg.de/hfm/CT/CT.html

${ }^{4}$ see magic.mpp.mpg.de/

${ }^{5}$ see www.iac.es/eno.php?op1=4\&lang=en and www.iac.es/proyecto/ site-testing/

${ }^{6}$ see www.iac.es/eno.php?op1=3

${ }^{7}$ see izana.aemet.es/index.php?lang=en
}

The atmosphere in the sub-tropical region of the $\mathrm{Ca}$ nary Islands is characterized by great stability throughout all the year. This is due to the combination of two processes of large-scale atmospheric circulation [12]: the descending branch of the Hadley cell around $30^{\circ} \mathrm{N}$ and the "Trade" or "Alisios" winds, coming from the Azores high area, that blow in the low troposphere, above an ocean which is relatively cold. As a result, a temperature inversion layer (TIL) appears above $1300 \mathrm{~m}$ a.s.l. in Winter $(\sim 1000 \mathrm{~m} \text { a.s.l. in Summer })^{8}$ [13], which can be usually well identified by the "sea of stratocumulus" at its base. This layer separates two well-defined regimes: below it, the moist marine boundary layer (MBL) and above it, the dry free troposphere (FT). The TIL is present in $>84 \%$ of the time in the Western Canary Islands ( $>90 \%$ observed at Guimar, Tenerife; the closest sounding station to $\mathrm{La}$ Palma), and shows moderate seasonal variation, with more frequent break-downs observed in February and November, probably modulated by the subsidence of the Hadley cell [13].

The ORM is located at an altitude between $2000 \mathrm{~m}$ and $2400 \mathrm{~m}$ a.s.l., hence well above the thermal inversion layer. This ensures that the installations are in the FT. At the ORM, clean air and clear sky conditions are hence prevailing all around the year. The reason is, by the way, twofold: apart from being located above the stable inversion layer, it is situated on an island far away from any significant industrial activity.

Now that site selection is accomplished and expected average observational duty cycle and performance established [14], the CTA-N is primarily interested in assessing molecular density profiles up to about $25 \mathrm{~km}$ a.s.l. and their variations, in understanding aerosol profiles and their optical characteristics, in order to trim its atmospheric calibration strategy [15].

\footnotetext{
${ }^{8}$ About $25 \%$ of the time, additional TIL's are present above the primary one mentioned here [13].
} 


\section{Molecular density profiles}

We studied 3 years (2013-2015) of now-cast predictions of the "Global Data Assimilation System" (GDAS) ${ }^{9}$, available in time steps of 3 hours UTC for La Palma, more specifically for the grid point $29^{\circ} \mathrm{N}, 18^{\circ} \mathrm{W}$, about $28 \mathrm{~km}$ northwards the ORM, above the Atlantic, smoothly joint with the NRLMSISE-00 data ${ }^{10}$, for lat. $28.77^{\circ}$, lon. $342.11^{\circ}$ above $20 \mathrm{~km}$ a.s.1. A spatially better resolved WRF model [16], as employed by J. Marín in these proceedings [17], has not yet been tried, however the European ECMWF ${ }^{11}$ is currently being tested, yielding similar or even better results, while the IG2 model [19] does not agree well with instantaneous MAGIC data at these altitude ranges, at least what concerns the temperature profiles. Similarly, the NRLMSISE-00 model [20], although very accurate above $20 \mathrm{~km}$ a.s.l., is not able to resolve all tropospheric temporal variations and can show deviations from the GDAS model of up to $10 \%$.

The GDAS data set is available in 24 pressure levels $^{12}$, each associated with an individual geopotential height, a temperature and other data, such as wind components and humidity. Geopotential heights have been converted to local altitudes a.s.1. using the WGS-84 ellipsoid for the evaluation of the combined effects of gravitational and centrifugal forces on the local gravity at a given altitude and latitude, following the prescription of Mahoney [21]. Values between these levels were interpolated. One has to keep in mind however, that fine-structures possibly present in the lower troposphere, and particularly in the tropopause (see e.g. Fig. 1 of [13] or Fig. 1 of [22]), cannot be reproduced by such an interpolated coarse grid.

For the purpose of ground validation, temperature and pressure values measured by the MAGIC weather station were compared with the predicted ones at the altitude of the weather station, which was assessed by a specialized company using a GPS survey in 2015. Fig. 1 shows all entries obtained for night-times. For the pressure comparison a bias of $(-0.25 \pm 0.01)$ mbar was obtained, compatible with no bias, once the accuracy of the MAGIC pressure sensor (of $\pm 0.8 \mathrm{mbar}$ ) is taken into account. The temperature comparison yields a significant bias of $(2.08 \pm 0.02)^{\circ} \mathrm{C}$, which can however be understood by ground cooling effects, that are not present at the chosen GDAS grid point (lying above the Sea). Using only day-time data, an opposite effect is observed. Typically such ground effects are limited to low altitudes. Nevertheless, even in the exaggerated assumption of being constant throughout the troposphere, this temperature bias would alter the density profile by only $0.8 \%$. Another direct validation was performed with the MAGIC LIDAR data, which shows pure molecular back-scattering and extinction signatures throughout most of the probed altitude ranges, and where

\footnotetext{
${ }^{9}$ https://www.ncdc.noaa.gov/data-access/model-data/ model-datasets/global-data-assimilation-system-gdas

${ }^{10} \mathrm{ccmc}$.gsfc.nasa.gov/modelweb/atmos/nrlmsise00.html

${ }^{11} \mathrm{http} / / /$ weather.unisys.com/ecmwf/index.php

${ }^{12} 1000,975,950,925,900,850,800,750,700,650,600,550,500$, $450,400,350,300,250,200,150,100,50,20 \mathrm{mbar}$, and the surface level.
}

contemporaneous GDAS profiles yield accurate $\chi^{2}$-fit distributions, in contrast to average molecular profiles [23].
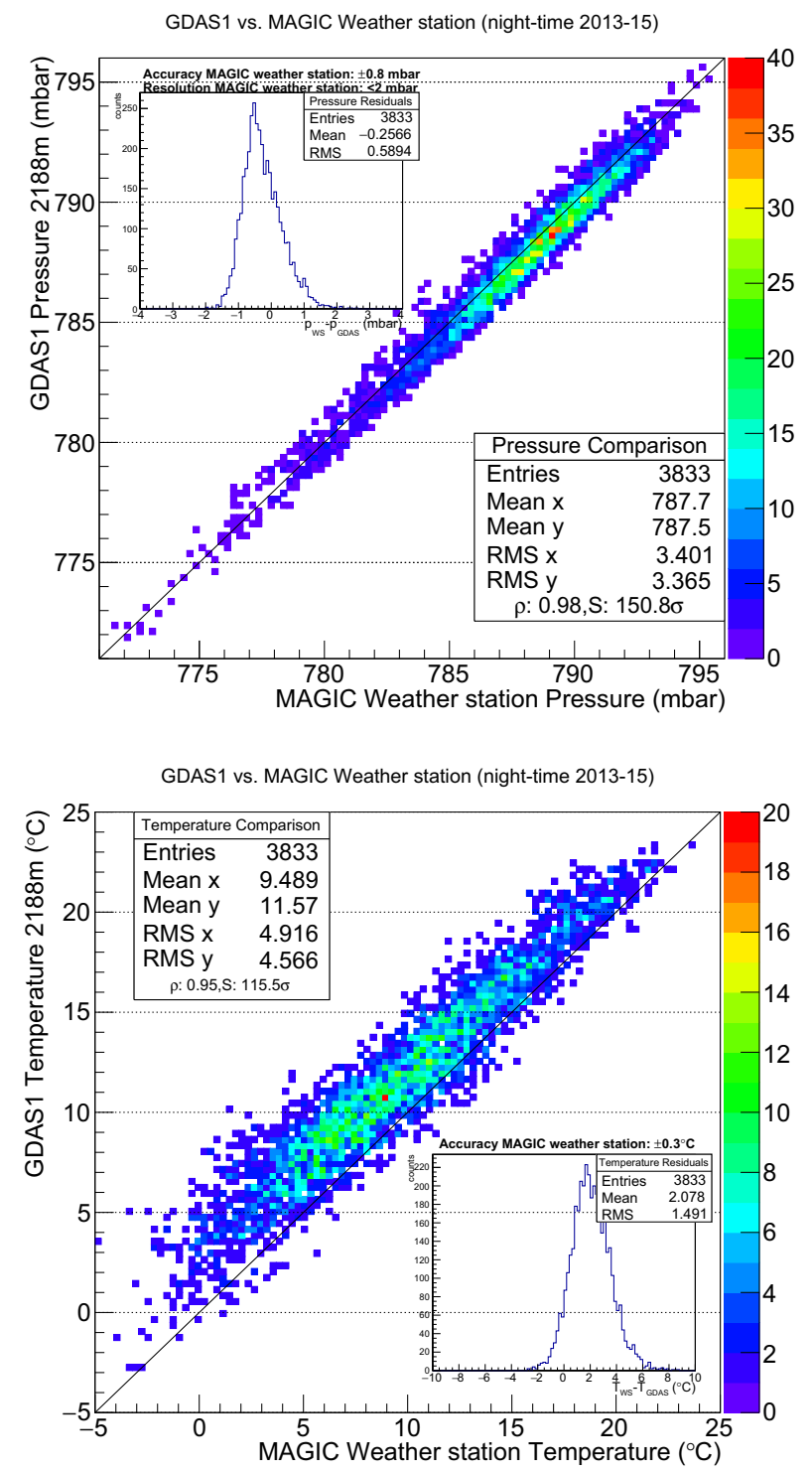

Figure 1. Cross-correlation of the GDAS predicted pressures and temperatures at $21 \mathrm{~h}, 0 \mathrm{~h}, 3 \mathrm{~h}$ and $6 \mathrm{~h}$ UTC at the altitude of MAGIC, with the simultaneous MAGIC weather station data. The inlets display the distribution of the respective differences.

Fig. 2 shows averaged density profiles, grouped according to the findings of [22]: an "average winter" (DEC,JAN,FEB,MAR,APR), characterized by multiple thermal tropopauses, which are several kilometers thick and can reach extremely high altitudes of up to $20 \mathrm{~km}$ a.s.l.. This typical mid-latitude tropopause is influenced by the sub-tropical jet stream (STJ) centered at lower latitudes. The "average summer" (JUL,AUG,SEP) model is characterized by only one thermal tropopause, typical for a tropical upper troposphere, with the STJ having moved northwards of the Canary Islands. The intermediate months (MAY,JUN,OCT,NOV) show transition behavior between both scenarios. Tropical tropopauses occur nevertheless also occasionally during the rest of the year. The lower end of the multi-layered, thick mid-latitude tropopauses move slightly upwards during spring and de- 
scend again in autumn, while the single-layered tropical tropopauses show much less seasonality [22]. From these average scenarios, and the extremes also seen in Fig. 2, the input models for detailed simulations have been created [24].

The most significant differences between both averages are found below $5 \mathrm{~km}$ a.s.l. and in the tropopause. The latter has also been observed in the South [17]. At about $15 \mathrm{~km}$, the strongest relative differences between Summer and Winter is observed, with an average seasonal variation amplitude of about $7 \%$ (see Fig. 2 bottom). Diurnal variations are more than an order of magnitude smaller at all altitudes above CTA-N.
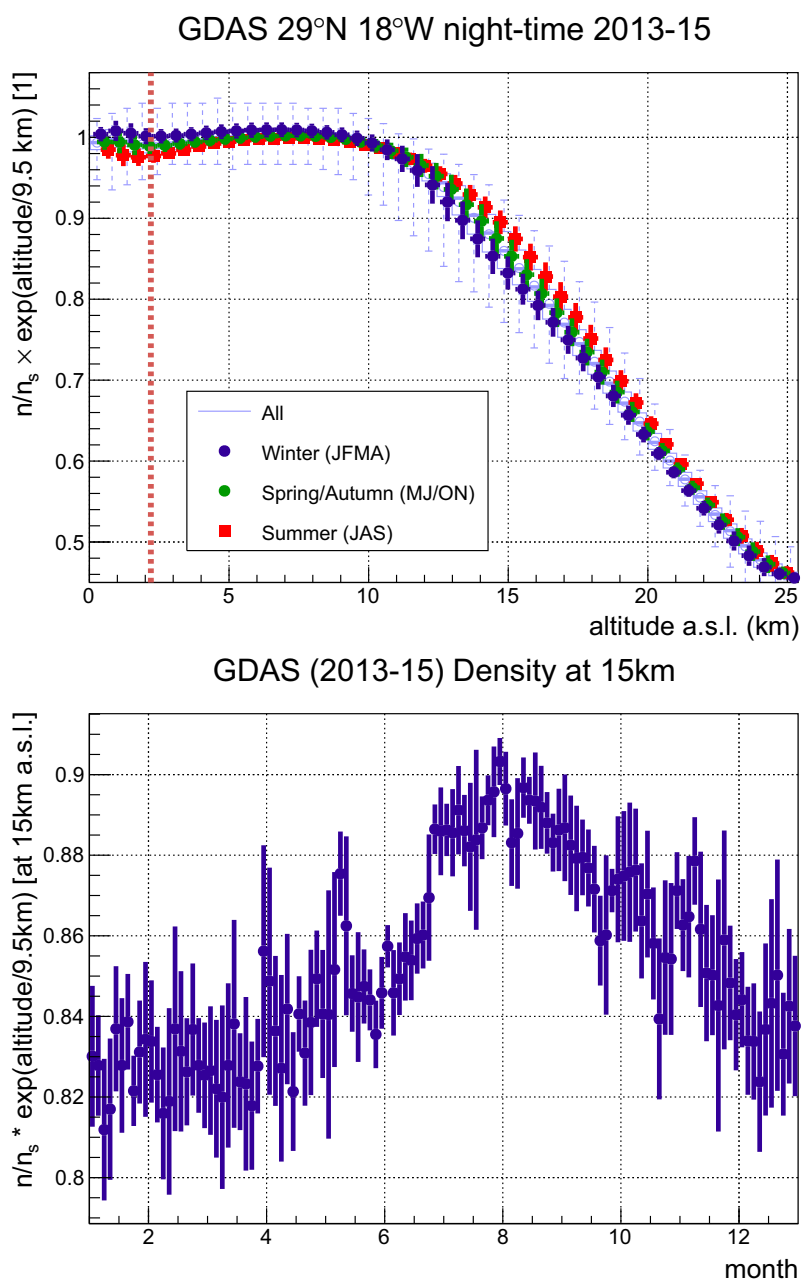

Figure 2. Top: density profiles of 3-years GDAS nowcasts at $21 \mathrm{~h}, 0 \mathrm{~h}, 3 \mathrm{~h}$ and $6 \mathrm{~h}$ UTC, scaled to the U.S. Standard Atmosphere density $n_{s}=2.55 \cdot 10^{19} \mathrm{~cm}^{-3}$ and multiplied with the exponential of an average scale height $\left(H_{s}=9.5 \mathrm{~km}\right)$ for better visibility. The dark blue, red and green points display an "average Winter", "average Spring/Autumn" and "average Summer" scenario, respectively. Error bars represent the standard deviation, while the light blue lines show the peak-to-peak differences for the entire data set. Bottom: seasonal variation of density evaluated at $15 \mathrm{~km}$ a.s.l.

Ozone intrusions into the upper troposphere, the "stratosphere-troposphere exchange" (STE) processes, have not been found for the Western Canary Islands, as assessed climatologies of the "Potential Vorticity" (PV) parameter [18]. Nevertheless, a 20 yr ozone-sonde campaign revealed that the extra-tropical tropopause above Tenerife shows increased ozone concentration during late Winter and Spring in the range from 12 to $15 \mathrm{~km}$ a.s.l. [22], well below the stratospheric ozone layer above $\sim 18 \mathrm{~km}$.

\section{Aerosol Profiles}

Aerosols have been characterized at the ORM with the help of elastic LIDARs [10, 23], dust counters [6] and extinction measurements by optical telescopes [8]. Moreover, several types of particulate analyzers and nephelometers are employed at the Izaña station on Tenerife [25-27] and on airplanes [28]. Sun photometer inversion products are available for Izaña through the AERONET network ${ }^{13}$. Also satellite information has been investigated [11] and back-trajectories simulated, using the HYSPLIT code [10, 29].

Ground-layer aerosol at the Western Canary Islands, of marine/oceanic origin with small admixture of anthropogenic aerosol [10] typically shows a bi-model size distribution during clear nights, with a size distribution peaking at $50 \mathrm{~nm}$ during night (see Fig. 7 of [25]), an exponential decay of density, with scale heights around $500 \mathrm{~m} \mathrm{[23]} \mathrm{and} \mathrm{aerosol} \mathrm{optical} \mathrm{thickness} \mathrm{(AOT)} \mathrm{of} \sim 0.02$ at $532 \mathrm{~nm}[10,23]$. In this situation, only Aitken and Accumulation modes of aerosol are found while the Coarse mode is practically absent. This leads to typical Ångström exponents of 1.5-2.0 [26, 30] and a single scattering albedo of 0.91 [27] and LIDAR ratios around $30 \mathrm{sr}$ [31]. These findings are compatible with the median value for the $V$-band extinction of 0.13 mag airmass $^{-1}$ found by [8], translating into an AOT of about 0.02.

The situation changes drastically during dust intrusion events from the Western Sahara (the so-called "calima"), when mean particle sizes reach $1 \mu \mathrm{m}$ or greater [6]. Such events occur during about $30 \%$ of the Summer nights [8], and rarely during the rest of the year. Typical LIDAR ratios are then $\sim 60$ sr [31], and AOTs can reach values in excess of 0.5 [6]. The calima-related dust can reach altitudes of and higher than $7 \mathrm{~km}$ a.s.l. [10], although lower altitudes are more frequent [23].

Fig. 3 shows the probability to observe a given value of AOT from different altitudes above the CTA-N, obtained with a 2-years statistics of the MAGIC LIDAR.

\section{Conclusions}

The atmosphere above the ORM has been characterized by a large variety of instruments in the past. For molecular and aerosol profiles, detailed climatological studies exist providing a deep understanding of their origins and characteristics. The CTA-N profits from these to design its atmospheric calibration strategy and reduce systematic uncertainties to hitherto unprecedented levels.

\footnotetext{
${ }^{13} \mathrm{http}$ //aeronet.gsfc.nasa.gov/cgi-bin/type_one_station_opera_v2_ inv2? site $=$ Izana $\&$ nachal $=0 \&$ level $=2 \&$ year $=-1 \&$ month $=0 \&$ day $=1 \&$ aero_water $=0 \&$ if_day $=0 \&$ if_err $=0 \&$ year_or_month $=0 \&$ place_code $=0$
} 


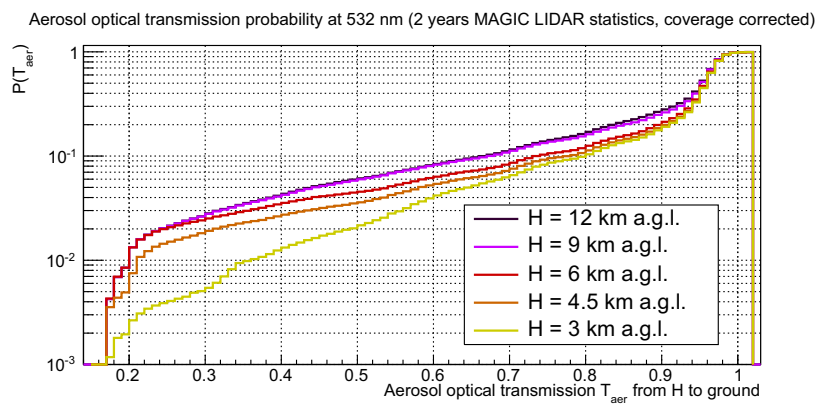

Figure 3. Aerosol Transmission probability for different emission altitude above the MAGIC LIDAR. Differences between the yellow line (emission height $3 \mathrm{~km}$ a.g.l.) and the red line (emission height $6 \mathrm{~km}$ a.g.1.) are mainly due to calima, whereas clouds are frequently found between $4.5 \mathrm{~km}$ and $9 \mathrm{~km}$ a.g.l.

\section{References}

[1] M. Actis et al., Design concepts for the Cherenkov Telescope Array CTA: an advanced facility for groundbased high-energy gamma-ray astronomy, Exp. Astron. 32, 193 (2011).

[2] C. Fruck et al., A novel LIDAR-based Atmospheric Calibration Method for Improving the Data Analysis of MAGIC, Proc. 33 ${ }^{\text {rd }}$ ICRC 1054 (2013), arXiv:1403.3591

[3] M. Gaug et al., Atmospheric Monitoring for the MAGIC Telescopes. Proc. $1^{\text {st }}$ AtmoHEAD Conf. (2014).

[4] C. Fruck \& M. Gaug, Atmospheric monitoring in MAGIC and data corrections, Proc. $2^{\text {nd }}$ AtmoHEAD Conf., EPJ 8902003 (2015).

[5] G. Lombardi et al., El Roque de Los Muchachos Site Characteristics. II. Analysis of Wind, Relative Humidity, and Air Pressure. PASP 119292 (2007).

[6] G. Lombardi et al., El Roque de Los Muchachos site characteristics. III. Analysis of atmospheric dust and aerosol extinction A\&A 483651 (2008).

[7] C. Muñoz-Tuñón et al., Night-time image quality at Roque de los Muchachos Observatory. Astron. Astrop. Suppl. Ser. 125 (1) 183 (1997).

[8] A. García-Gil et al., Atmosphere Extinction at the ORM on La Palma: A 20 yr Statistical Database Gathered at the Carlsberg Meridian Telescope. PASP 122 1109 (2010).

[9] B. García-Lorenzo et al. Infrared astronomical characteristics of the Roque de los Muchachos Observatory: precipitable water vapour statistics MNRAS 405 (4) 2683 (2010).

[10] M. Sicard et al., Results of site testing using an aerosol, backscatter lidar at the Roque de los Muchachos Observatory MNRAS 405 (1) 129 (2010).

[11] A. M. Varela et al., Astronomical site selection: on the use of satellite data for aerosol content monitoring MNRAS 391 (2) 507 (2008).

[12] E. Palmen \& C. W. Newton. Atmospheric Circulation Systems - Their Structure and Physical Interpretation Intern. Geophys. Ser. 13 Acad. Press (1969).

[13] J. Carrillo et al., Characterization of the marine boundary layer and the trade-wind inversion over the sub-tropical North Atlantic Boundary-Layer Meteor. 158 (2) 311 (2016).

[14] T. Hassan et al., Monte Carlo Performance Studies of Candidate Sites for the Cherenkov Telescope Array, subm. to Astrop. Phys. (2017).

[15] M. Gaug, CTA Atmospheric Calibration, these proc.

[16] W. C. Skamarock et al., A Description of the Advanced Research WRF NCAR/TN-468+STR (2008).

[17] J. Marín et al., Density profile characterization and modeling at Paranal and Armazones $2 k$ sites, these proc.

[18] E. Cuevas et al., Surface O3 characterization in the sub-tropical North Atlantic troposphere, Atmos. Chem. Phys. 131973 (2013).

[19] J. J. Remedios et al., MIPAS reference atmospheres and comparisons to V4.61/V4.62 MIPAS level 2 geophysical data sets Atm. Chem. Phys. Disc. 79973 (2007).

[20] J. M. Picone et al., NRLMSISE-00 empirical model of the atmosphere: Statistical comparisons and scientific issues. J. Geophys. Res.: Space Phys. 107 (A12) SIA 151 (2002).

[21] M. J. Mahoney. A discussion of various measures of altitude, NASA Jet Propulsion Laboratory, http://mtp.mjmahoney.net/www/notes/altitude/ altitude.html (2001).

[22] J. J. Rodriguez-Franco \& E. Cuevas, Characteristics of the subtropical tropopause region based on longterm highly resolved sonde records over Tenerife. J. Geophys. Res.: Atmospheres 118 (19) 10754 (2013).

[23] C. Fruck et al., Characterizing the atmosphere over the MAGIC site, analyzing LIDAR data with a novel signal inversion algorithm, in preparation.

[24] M. Vrastil et al., Overview of Atmospheric Simulation Efforts in CTA, these proc..

[25] S. Rodríguez et al., Atmospheric nanoparticle observations in the low free troposphere during upward orographic flows at Izaña Mountain Observatory Atmos. Phys. Chem. 96319 (2009).

[26] H. Maring et al., Aerosol physical and optical properties and their relationship to aerosol composition in the free troposphere at Izaña, Tenerife, Canary Islands, during July 1995, J. Geophys. Res. 10514677 (2000).

[27] E. Andrews et al. Climatology of aerosol radiative properties in the free troposphere Atmosph. Res. 102 365 (2011).

[28] D. R. Collins et al. In situ aerosol-size distributions and clear-column radiative closure during ACE-2 Tellus 52B 498 (2000).

[29] S. Rodríguez et al. Transport of desert dust mixed with North African industrial pollutants in the subtropical Saharan Air Layer Atmos. Chem. Phys. Disc. 11 8841 (2011).

[30] D. Whittet et al., The extinction properties of Sahara dust over La Palma Vistas in Astronomy 30135 (1987).

[31] A. Ansmann \& D. Müller, LIDAR Range-Resolved Optical Remote Sensing of the Atmosphere, p. 112, Springer, Berlin (2005). 